\title{
The prevalence of burnout among registrars in the School of Clinical Medicine at the University of the Witwatersrand, Johannesburg, South Africa
}

\author{
C Zeijlemaker, MD (Amsterdam), FCFP (SA), MMed; S Moosa, MB ChB, MFamMed, MBA, PhD \\ Division of Family Medicine, Department of Family Medicine and Primary Care, School of Clinical Medicine, Faculty of Health Sciences, \\ University of the Witwatersrand, Johannesburg, South Africa
}

Corresponding author: C Zeijlemaker (cathelijn.zeijlemaker@gmail.com)

\begin{abstract}
Background. Burnout is a response to prolonged stress and consists of three elements: emotional exhaustion (EE), depersonalisation (DP), and feelings of personal accomplishment (PA). The existence of burnout in doctors is often not acknowledged but has major consequences for personal and professional life. Only limited research has been done on the prevalence of burnout among registrars in South Africa (SA). Objectives. To describe the prevalence of burnout in a cohort of SA registrars, and assess relationships between burnout and sociodemographic factors.

Methods. A cross-sectional descriptive internet survey was conducted. Respondents were registrars in departments of the School of Clinical Medicine at the University of the Witwatersrand, Johannesburg, SA. The Maslach Burnout Inventory (MBI) was used to measure burnout. Relationships were assessed by the independent-samples $t$-test and analysis of variance.

Results. A total of 585 emails were delivered to registrars, of whom 201 started the survey (response rate $34 \%$ ); 170 questionnaires were analysed. The mean age of the respondents was 33 years, and the male/female ratio was 1:1.8. The mean (standard deviation) score for EE was 3.5 (1.2), for DP 2.7 (1.1) and for PA 4.1 (1.1). The overall level of burnout was $84 \%$. None of the respondents scored low over all categories. No significant association between sociodemographics (age, sex, discipline, year in the programme and experience) and MBI dimensions was found.

Conclusions. The prevalence of burnout in this study was higher than that reported in the national and international literature. Levels of DP were extremely high and are worrying, as DP affects professionalism and engagement of doctors. In keeping with the literature, no associations were found between sociodemographic factors and burnout, suggesting that the cause of burnout should be sought in the work environment. Efforts to improve autonomy in the workplace, development opportunities and promoting peer collaboration are needed to prevent burnout.

S Afr Med J 2019;109(9):668-672. https://doi.org/10.7196/SAMJ.2019.v109i9.13667
\end{abstract}

Medical doctors are at high risk of burnout compared with other professionals. ${ }^{[1]}$ At the onset of medical training their mental health profiles are similar to those of their peers, but through medical school into registrar training there is a progressive downward trend. ${ }^{[2]}$ In the USA, $60 \%$ of registrars suffered burnout in $2014 .{ }^{[2]}$ In South Africa (SA), limited research has been done on the prevalence of burnout among registrars.

Burnout is a response to prolonged occupational stress and consists of three different elements that may coexist to varying degrees: (i) emotional exhaustion (EE), the feeling that work is overwhelming and energy levels are depleted; (ii) depersonalisation (DP), a personal detachment from work, resulting in unfeeling and impersonal responses towards colleagues and patients; and (iii) personal accomplishment (PA), low scores indicating feelings of inefficacy, incompetence and lack of personal achievement. This is the theoretical framework most commonly used in the literature on burnout and informs the Maslach Burnout Inventory (MBI) tool. ${ }^{[3]}$

The existence of burnout is often not acknowledged. Medical doctors have poor insight into their symptoms, and find it difficult to admit to weaknesses and play the patient role. Not recognising the existence of burnout leads to poor coping strategies and help-seeking behaviour. ${ }^{[3,4,5]}$

The consequences of burnout are well studied and affect both personal and professional life. Studies on the impact of burnout on personal life have found associations with alcohol abuse and dependence. Suicidal thoughts and ideation are more common among doctors with burnout than among those without. Other personal effects of burnout manifest in increased relationship stress and motor vehicle accidents. ${ }^{[6]}$

Burnout affects a range of qualities associated with professional development: concentration, honesty, integrity, empathy, altruism and self-regulation. ${ }^{[6]}$ The literature suggests that deficits in these areas may increase the risk of medical errors and poor medical care. ${ }^{[7,8]}$ Furthermore, burnout affects the cognitive processes needed to gain knowledge and skills. A study by West et al..$^{[9]}$ testing medical knowledge of internal medicine registrars in the USA showed significantly lower results in subjects with burnout. ${ }^{[9]}$ Lastly, burnout negatively influences the attitude of registrars to their careers, leading to changes of specialty, changes of career or emigration. ${ }^{[6]}$ In SA, these effects can be detrimental to the existing compromised medical workforce.

Looking at the causes of burnout, a reason why registrars specifically can be affected by burnout may be found in the demandcontrol-support model. This model explains how burnout from work-related stress is triggered. If workload is increased while individual autonomy at work is decreased, work stress starts to affect the registrar's personal life. ${ }^{[10]}$

Associations between demographics and burnout could be expected, but so far studies have failed to prove them. ${ }^{[10]}$ Although women have a higher lifetime risk of depression than men, a review 
of studies done on registrar burnout has showed no difference in the prevalence of burnout between genders. ${ }^{[10]}$ Another possible cause of burnout may lie in the discipline in which the registrar is specialising. One study found that doctors working in the front line of service (family medicine, emergency medicine, internal medicine and neurology) had an increased prevalence of burnout. ${ }^{[1]}$ Whether this difference in discipline-associated burnout can be extrapolated to registrars has not been studied. Lastly, stress is a continuous process, from graduation (and before) onwards; higher levels of burnout could therefore be expected among doctors with more years' postqualification experience, or registrars who have spent a longer time in their programme.

Literature on the prevalence of burnout among SA registrars is limited. Studies in Western Cape and Free State provinces have shown similar severe levels of burnout in doctors; however, in the Western Cape only a few registrars participated. ${ }^{[11-13]}$ In Gauteng, emergency medicine registrars had significantly higher levels of burnout than specialists and medical officers in the same departments. ${ }^{[14]}$ Registrars in anaesthetics in Gauteng were also found to have higher levels of burnout than medical officers and consultants, but this was not statistically significant. ${ }^{[15]}$ Despite a small sample size, a study in an obstetrics and gynaecology department also suggested high burnout levels among registrars. ${ }^{[16]}$

\section{Objectives}

Registrars are the future specialists in our healthcare system, and burnout will affect this new generation in terms of both quality and quantity. It is therefore of great importance to improve understanding of burnout among registrars. The objectives of this study were to explore the prevalence of burnout among registrars training in the School of Clinical Medicine at the University of the Witwatersrand, and to determine sociodemographic factors associated with burnout.

\section{Methods \\ Design}

The study design was cross-sectional and descriptive.

\section{Setting/study population}

Email addresses were obtained for a study population of 759 registrars in nine departments: Family Medicine, Paediatrics, Surgery, Internal Medicine, Radiation Sciences, Neurosciences, Anaesthesia, Obstetrics and Gynaecology and Psychiatry. Supernumerary registrars were excluded from the study. The initial sample size was estimated at 170 , with a $95 \%$ confidence interval and the assumptions of $5 \%$ precision and an estimated $80 \%$ prevalence. ${ }^{[2,11,12]}$ However, all emails were sampled owing to the predicted low response rate for internet surveys. ${ }^{[17]}$ A total of 585 emails were delivered to the 759 addresses (the others went to incorrect addresses or bounced back). Of 585 registrars whose emails were delivered, 201 started the survey (the others may not have had active accounts, or may not have read the email), giving a response rate of $34 \%$. Two reminder emails were sent out 4 days apart. The study was closed once the sample target of 170 was reached.

\section{Data collection}

The questionnaire consisted of two parts. The first part contained questions on sociodemographics: gender, age, clinical discipline, years since graduation, and years in the programme. The second part consisted of the MBI. The MBI is an instrument to measure burnout and is the most validated and widely used survey tool; according to the literature, its reliability, using Cronbach's alpha, is $0.71-0.9 .^{[18]}$ The MBI consists of a 22-item questionnaire. The 22 questions are answered using a 7-point scale from 0 (never) to 6 (very often), to reflect the frequency with which certain feelings and responses are experienced. The 22 questions assess and score the three aspects of burnout, i.e. EE, DP and PA. ${ }^{[18]}$

In clinical practice, the MBI score should not be seen as a dichotomous variable, meaning that burnout is either present or not present. Maslach, the developer of the MBI tool, has therefore moved away from using cut-off scores to define burnout. However, cut-off scores are needed for research purposes. Review of the literature shows that $79.5 \%$ of studies used $\geq 27$ as a cut-off score for high EE. ${ }^{[19]}$ For DP the recommendation is set at 10, although scores varying between 9 and 30 can be used. ${ }^{[19]}$ In the present study, the recommendations in the Maslach guidelines were used (Table 1). ${ }^{[18]}$ Respondents with burnout are defined as having a high score in either the EE category or the DP category. In the EE and DP category, higher scores are an indication of a worse level of burnout, whereas a higher score in PA correlates with a lower level of burnout and is regarded as protective against burnout.

The email invitation contained information regarding the study. However, to avoid sensitisation to burnout, the email did not use the term 'burnout'; instead, 'work-related feelings affecting psychological wellbeing' was used. The information leaflet gave more detailed background on the purpose of the study. Respondents were required to consent before proceeding with the online questionnaire. Participation was anonymous. Administration instructions for the questionnaire tool were provided to reduce response bias. ${ }^{[18]}$ The study facilitator was a registrar, and so an equal to the respondents. Respondents were requested to provide an email address if they wanted feedback on their scores. In addition, no identifiers were captured in order to ensure confidentiality. By motivating the respondents to complete the questionnaire in a private environment, privacy would be ensured, which reduced social desirability bias. The software programme (Qualtrics, Provo, UT) used, compatible with different devices, including smart phones, facilitated this.

On completion of the questionnaire, information about psychological support from Student Services was provided. Reference to a general article about resilience training was also provided. ${ }^{[20]}$ Respondents could request to be informed by the researcher of their individual burnout score and its meaning. The Human Research Ethics Committee of the University of the Witwatersrand granted permission to conduct the study (ref. no. M161033). Permission to use the MBI tool was granted by the patent holders.

\section{Statistical analysis}

A statistician assisted with the data analysis. Data were transferred from Qualtrics (Provo, UT) and analysis was carried out using SAS version 9.4 for Windows (SAS, USA) and SPSS Statistics for Windows, version 25 (IBM, USA). The 5\% significance level was used throughout. Scores for each of the three MBI dimensions were created on the scale of the original questions $(0-6)$ by dividing the total score for each dimension by the number of questions contributing to that dimension. Age and years since graduation were categorised

Table 1. Classification of burnout according to the Maslach Burnout Inventory ${ }^{[18]}$

\begin{tabular}{|c|c|c|c|}
\hline Category & Low range & Average range & High range \\
\hline EE (9 questions) & $\leq 18$ & $19-26$ & $\geq 27$ \\
\hline DP (5 questions) & $\leq 5$ & $6-9$ & $\geq 10$ \\
\hline $\mathrm{PA}^{*}$ (8 questions) & $\geq 40$ & $39-34$ & $\leq 33$ \\
\hline
\end{tabular}


into approximate quartiles for between-group comparisons. Clinical discipline type was requested, but data analysis grouped disciplines according to poor response rates or cohorts with low numbers. The relationship between each MBI score and gender, age (categorised), clinical discipline, year in programme, and number of years since graduation (categorised) was assessed by the independent-samples $t$-test for two categories and one-way analysis of variance for more than two categories.

\section{Results}

A total of 170 questionnaires were analysed. The mean age of the respondents was 33 years (interquartile range (IQR) 29 - 37). There was a wide range of years since graduation from medical school ( 5 - 27 years). The average post-graduation experience was 9 years (IQR 6 - 12). Of the respondents, 64\% $(n=109)$ were female and $36 \%(n=61)$ were male (male/female ratio 1:1.8). Most departments in the School of Clinical Medicine were represented in the group of respondents. Best represented was Internal Medicine (21\%), followed by Paediatrics (14\%), Psychiatry (12\%) and Surgery (11\%). There was an interest in burnout among $86 \%$ of the respondents, as suggested by their requests for feedback. An overview of the sociodemographics is presented in Table 2.

The mean (standard deviation (SD)) score for EE was 3.5 (1.2, median 3.6), with a minimum average score of 0.2 and a maximum average of 6.0. The mean (SD) score for DP was 2.7 (1.1, median 2.7 ), with a minimum average score of 0.2 and a maximum average of 4.8. The mean (SD) score for PA was 4.1 (1.1, median 4.1), with a minimum average of 0.3 and a maximum average of 6.0 .

The distribution of respondents in terms of the MBI classification of burnout (Table 1) is shown in Table 3. According to the definition, with a high score in the EE or DP category, $84 \%$ of the registrars had burnout. Many of these registrars had scores indicating burnout in more than one of the categories: $41 \%(n=58)$ had high-risk scores in all categories, $23 \%(n=39)$ had high scores in both EE and DP, and $32 \%(n=46)$ had a high score in only EE or DP.

The opposite of burnout is engagement (i.e. low scores for $\mathrm{EE}$ and DP and a high score for PA). None of the participants had scores indicating engagement over all categories. The closest to engagement were 10 participants (6\%) who scored in the low range of burnout for PA in combination with low to moderate EE and DP.

The relationships between burnout and sociodemographics were analysed. The distribution of dimension scores across age, gender, discipline, programme year and postgraduate (PG) experience is shown in Table 4. There was no significant association between age, gender, discipline, year in the programme or PG experience and any of the three MBI dimensions. The $p$-values for the between-group differences are also shown in Table 4.

\section{Discussion}

Overall, a concerningly high burnout level of $84 \%$ was found. This is higher than rates in other studies. A 2012 survey of 1701 registrars in the USA found a burnout level of $60.3 \% .^{[2]}$ In Lebanon, a developing country, 155 medical registrars had a burnout level of $80 \% .{ }^{[18]}$ This figure may be a slight overestimate, however, as the authors' definition of burnout was less stringent than ours and defined the risk as high if a score in any category was high. ${ }^{[18]}$ Locally, similar and higher levels of burnout were found in smaller samples in departments of Emergency Medicine and Obstetrics and Gynaecology. ${ }^{[14,16]}$ High levels of burnout among registrars were also found in Free State Province, but because of different cut-off points per category, the results cannot be compared with ours. ${ }^{[12]}$ Demographically, studies in the Western Cape have shown that burnout levels were similar in rural and urban areas. ${ }^{[1,13]}$
Table 2. Sociodemographics of the study respondents $(N=170)$

\begin{tabular}{ll}
\hline Variables & $\boldsymbol{n}(\%)$ \\
\hline Sex & $109(64)$ \\
Female & $61(36)$ \\
Male & \\
Discipline & $15(8.8)$ \\
Anaesthesia & $1(0.6)$ \\
Cardiothoracic Surgery & $11(6.5)$ \\
Emergency Medicine & $15(8.8)$ \\
Family Medicine & $36(21.2)$ \\
Internal Medicine & $4(2.4)$ \\
Neurosurgery & $7(4.1)$ \\
Obstetrics & $1(0.6)$ \\
Ophthalmology & $8(4.7)$ \\
Orthopaedic Surgery & $1(0.6)$ \\
Paediatric Surgery & $23(13.5)$ \\
Paediatrics & $21(12.4)$ \\
Psychiatry & $9(5.3)$ \\
Radiology & $18(10.6)$ \\
Surgery & \\
Year in programme & $24(14.1)$ \\
1 & $45(26.5)$ \\
2 & $39(22.9)$ \\
3 & $38(22.4)$ \\
4 & $7(2.9)$ \\
5 & $1(0.6)$ \\
6 & $16(9.4)$ \\
Completed & 170 \\
Total &
\end{tabular}

Table 3. Maslach Burnout Inventory scores per category of the study respondents $(N=170)$

\begin{tabular}{llll}
\hline & Low, $\boldsymbol{n} \%$ & Medium, $\boldsymbol{n} \%$ & High, $\boldsymbol{n} \%$ \\
\hline $\mathrm{EE}$ & $24(14.1)$ & $33(19.4)$ & $113(66.5)$ \\
$\mathrm{DP}$ & $10(5.9)$ & $33(19.4)$ & $127(74.7)$ \\
$\mathrm{PA}^{*}$ & $89(52.4)$ & $43(25.3)$ & $38(22.4)$ \\
$\begin{array}{l}\text { EE = emotional exhaustion; DP = depersonalisation; PA = personal achievement. } \\
\text { *For EE and DP, higher scores are an indication of a worse level of burnout, whereas a } \\
\text { higher score in PA correlates with a lower level of burnout. }\end{array}$
\end{tabular}

Comparing the MBI dimension of EE of registrars in the present study with registrars abroad, the proportion of respondents with high scores (67\%) was similar to that among Lebanese registrars (67.7\%). ${ }^{[21]}$ These figures are much higher than those for registrars in the USA $(44.4 \%))^{[2]}$ As EE is largely related to the demands of work, it is interesting to see similarly high levels of EE in developing countries, where doctors may have to cope with more pressure than those in the developed world.

Of note is the very high proportion of respondents in the present study with high scores for DP (75\%). This figure is exceptionally high, as apart from the data from the Western Cape, with a DP level of $64 \%{ }^{[11]}$ other studies mentioned above have all reported levels in the range of $\leq 50 \%$. High DP is concerning, as this aspect of burnout reflects detachment and an impersonal response to patients and is associated with negative effects on professionalism. ${ }^{[7]}$

$\mathrm{PA}$ is recognised as protective against burnout, with high scores corresponding to less experienced burnout, and the $22 \%$ of registrars with PA scores indicating a low risk of burnout in the present study is comparable with figures in other training institutions. In contrast, $52 \%$ of registrars in this study had PA scores indicating a high risk of burnout, and data from other studies all show levels below this. ${ }^{[2,11-16]}$ 


\begin{tabular}{|c|c|c|c|}
\hline Variables & $\begin{array}{l}\text { EE, mean } \\
\text { (SD) }\end{array}$ & $\begin{array}{l}\text { DP, mean } \\
\text { (SD) }\end{array}$ & $\begin{array}{l}\text { PA, mean } \\
\text { (SD) }\end{array}$ \\
\hline \multicolumn{4}{|l|}{ Age (years) } \\
\hline $27-29$ & $3.2(1.1)$ & $2.7(1.2)$ & $4.1(1.2)$ \\
\hline $30-31$ & $3.4(1.2)$ & $2.9(1.1)$ & $4.2(1.1)$ \\
\hline $32-33$ & $3.6(1.2)$ & $2.8(1.1)$ & $3.8(1.0)$ \\
\hline $34-35$ & $3.5(1.1)$ & $2.6(1.0)$ & $4.1(1.0)$ \\
\hline$\geq 36$ & $3.5(1.2)$ & $2.5(1.0)$ & $4.4(0.9)$ \\
\hline Variance $p$-value & 0.67 & 0.57 & 0.13 \\
\hline \multicolumn{4}{|l|}{ Gender } \\
\hline Female & $3.6(1.1)$ & $2.6(1.1)$ & $4.0(1.1)$ \\
\hline Male & $3.3(1.2)$ & $2.9(1.0)$ & $4.1(1.1)$ \\
\hline Variance $p$-value & 0.095 & 0.19 & 0.19 \\
\hline \multicolumn{4}{|l|}{ Discipline } \\
\hline Anaesthetics & $3.6(1.3)$ & $2.5(1.1)$ & $3.6(1.3)$ \\
\hline Emergency Medicine & $3.4(1.3)$ & $3.2(1.0)$ & $4.3(1.3)$ \\
\hline Family Medicine & $3.7(1.2)$ & $2.8(1.1)$ & $4.4(0.9)$ \\
\hline Internal Medicine & $3.3(1.0)$ & $2.5(1.1)$ & $4.0(1.2)$ \\
\hline Obstetrics & $3.7(0.9)$ & $2.9(1.1)$ & $3.7(1.3)$ \\
\hline Orthopaedics & $3.9(1.2)$ & $2.8(1.1)$ & $4.4(0.8)$ \\
\hline Paediatrics & $3.7(1.2)$ & $2.8(1.1)$ & $4.1(0.9)$ \\
\hline Psychiatry & $3.3(1.4)$ & $2.4(1.1)$ & $4.6(0.9)$ \\
\hline Radiology & $3.2(1.4)$ & $2.8(0.7)$ & $3.9(0.9)$ \\
\hline Surgery group ${ }^{*}$ & $3.4(1.1)$ & $2.9(1.1)$ & $3.8(0.9)$ \\
\hline Variance $p$-value & 0.90 & 0.63 & 0.12 \\
\hline \multicolumn{4}{|l|}{ Programme year } \\
\hline 1 & $3.1(1.1)$ & $2.8(1.1)$ & $3.9(1.0)$ \\
\hline 2 & $3.5(1.1)$ & $2.7(1.2)$ & $4.0(1.2)$ \\
\hline 3 & $3.5(1.2)$ & $2.7(1.1)$ & $4.0(1.0)$ \\
\hline 4 & $3.7(1.2)$ & $2.7(1.0)$ & $4.2(0.9)$ \\
\hline 5 & $3.4(1.2)$ & $2.7(1.2)$ & $3.6(1.1)$ \\
\hline 6 & $3.4(1.5)$ & $2.6(0.9)$ & $4.6(0.8)$ \\
\hline Variance $p$-value & 0.64 & $>0.99$ & 0.19 \\
\hline \multicolumn{4}{|l|}{ PG experience (years) } \\
\hline $1-6$ & $3.3(1.2)$ & $2.9(1.3)$ & $4.2(1.3)$ \\
\hline $7-8$ & $3.5(1.1)$ & $2.8(1.0)$ & $4.1(1.0)$ \\
\hline $9-10$ & $3.6(1.3)$ & $2.7(1.1)$ & $3.9(1.1)$ \\
\hline $11-12$ & $3.7(1.0)$ & $2.7(0.9)$ & $3.9(0.9)$ \\
\hline$\geq 13$ & $3.2(1.4)$ & $2.4(1.1)$ & $4.5(0.8)$ \\
\hline Variance $p$-value & 0.63 & 0.61 & 0.21 \\
\hline
\end{tabular}

Similar to studies elsewhere, in the present study burnout was not associated with sociodemographic factors such as age, sex, discipline, year in the programme and experience. This finding suggests that the causes of burnout lie in contextual factors such as organisational and management structures in the work environment.

As mentioned above, according to the demand-control-support model, burnout is caused by an imbalance of demands and resources in the work setting. EE has been found to be directly associated with increased demands. The very high levels of EE among registrars in the present study could indicate that unreasonably high demands are placed upon registrars. It has been suggested by Dyrbye and Shanafelt ${ }^{[6]}$ that these increased demands could be triggered by changes in the healthcare system, workforce shortages, 'curriculomegaly' and increased competition for registrar posts.

Poor levels of PA are closely related to lack of available resources. The conservation of resources theory links PA to resources in the workplace. If demands and resources are in balance, the system will function optimally. In view of the high scores for EE in the present study, an intervention could be to ensure that resources are sufficient to effectively meet the increased demands. Some of the resources to increase in PA are autonomy, development opportunities and job variety. ${ }^{[3]}$ Professional autonomy involves control over the terms and content of work, and has been shown to increase doctors' work satisfaction. ${ }^{[22]}$ Job variety could be addressed by giving opportunities to do different rotations within the registrar programmes. Focus on these aspects in current registrar training curricula could prevent burnout.

Further ways to target high levels of burnout could include both individual and group efforts. For the individual, positive results have been achieved using mindfulness-based interventions, ${ }^{[23]}$ for example through meditation, cognitive or behavioural therapy. Systemic efforts could come from management level at the training institutions. An intervention that has been shown to be successful is the CREW method (civility, respect, and engagement at work) in which a more civil working atmosphere is created by means of facilitations in regular workshops to create awareness of social behaviour. In this intervention, the focus is on improving the working relationship between colleagues. The CREW method showed that peer collaboration in the learning environment contributed to the prevention of burnout. ${ }^{[24]}$ Besides preventing burnout, better collaboration and teamwork are also likely to benefit general healthcare outcomes.

Recognition of the magnitude of burnout, as shown in this study, should make policy makers aware of their responsibility to find and implement solutions to this epidemic affecting the new generation of specialists. By targeting contextual factors to improve professional autonomy, job variety, and development opportunities, as well as individual factors promoting team collaboration, burnout could be prevented.

\section{Study limitations}

A limitation of this study is the sample bias. Although the actual response rate was much higher than expected, a non-probability convenience sample was used to achieve a sufficient sample size. A large percentage of the email addresses provided by the departments were incorrect or inactive. It is unclear how many of the nonresponders did not read the email, or perhaps were not interested in the topic. This method of sampling could have affected the epistemological validity of the results.

\section{Conclusions}

Overall, a concerningly high burnout level of $84 \%$ was found. This figure is higher than those in other national and international studies. High EE is associated with high work demands, which may particularly affect registrars training in developing countries. The extremely high level of DP is worrying, as this affects professionalism and negatively affects engagement of doctors with their patients. The cause of burnout should be sought in the work environment, as, in keeping with international literature, no associations with sociodemographic factors were found. PA is protective of burnout. Improving professional autonomy, job variety and development opportunities could help prevent burnout. Efforts should be made to improve peer collaboration, as this also assists in preventing burnout. 
Declaration. Publication of this research report was a requirement for CZ's MMed degree in Family Medicine.

Acknowledgements. We thank P Gaylard for analytical support, and H Potgieter and Prof. M Lukhele of the School of Clinical Medicine for their assistance conducting this research.

Author contributions. SM contributed to this article as CZ's MMed supervisor.

Funding. None.

Conflicts of interest. None.

1. Shanafelt TD, Boone S, Tan L, et al. Burnout and satisfaction with work-life balance among US physicians relative to the general US population. Arch Intern Med 2012;172(18):1377-1385. https://doi.org/10.1001/ rchinternmed.2012.3199

2. Dyrbye LN, West CP, Satele D, et al. Burnout among U.S. medical students, residents, and early career physicians relative to the general U.S. population. Acad Med 2014;89(3):443-451. https://doi.org/10.1097/ ACM. 0000000000000134

3. Maslach C, Jackson SE, Leiter MP. Maslach Burnout Inventory: Third edition. In: Zalaquett CP, Wood RJ, eds. Evaluating Stress: A Book of Resources. Lanham, Md: Rowman \& Littlefield Publishers, 1997:191-218. 4. Ellis C. Doctors get depressed too. S Afr Med J 2002;9(9):700.

5. Pretorius D, Basson WJ, Ogunbanjo GA. Personality profile and coping resources of family medicine vocational trainees at the University of Limpopo, South Africa. S Afr Fam Pract 2010;52(5):446-450. https://doi.org/10.1080/20786204.2010.10874023

6. Dyrbye L, Shanafelt TD. A narrative review on burnout experienced by medical students and residents. Med Educ 2015;50(1):132-149. https://doi.org/10.1111/medu.12927

7. Shanafelt TD, Bradley K, Wipf JE, et al. Burnout and self-reported patient care in an internal medicine residency program. Ann Intern Med 2002;136(5):358-367. https://doi.org/10.7326/0003-4819-136-5200203050-00008

8. West $\mathrm{CP}$, Tan $\mathrm{AD}$, Habermann TM, et al. Association of resident fatigue and distress with perceived medical errors. JAMA 2009;302(12):1294-1300. https://doi.org/10.1001/jama.2009.1389

9. West CP, Shanafelt TD, Kolars, JC. Quality of life, burnout, educational debt, and medical knowledge among internal medicine residents. JAMA 2011;306(9):952-960. https://doi.org/10.1001/jama.2011.1247

10. Niku K, Thomas MD. Resident burnout. JAMA 2004;292(23):2880-2889. https://doi.org/10.1001/ jama.292.23.2880

11. Rossouw L, Seedat S, Emsley RA, et al. The prevalence of burnout and depression in medical doctors working in the Cape Town Metropolitan Municipality community healthcare clinics and district hospitals of the Provincial Government of the Western Cape: A cross-sectional study. S Afr Fam Pract 2013;55(6):567-573. https://doi.org/10.1080/20786204.2013.10874418
12. Sirsay U, Steinberg WJ, Raubenheimer JE. Levels of burnout among registrars and medical officers working at Bloemfontein public healthcare facilities in 2013. S Afr Fam Pract 2016;58(6):213-218. working at Bloemfontein public healthcare facitic
https://doi.org/10.1080/20786190.2016.1198088

13. Liebenberg AR, Coetzee JF Jr, Conradie HH, Coetzee JF. Burnout among rural hospital doctors in the Western Cape: Comparison with previous South African studies. Afr J Prim Health Care Fam Med 2018;10(1):a1568. https://doi.org/10.4102/phcfm.v10i1.156

14. Rajan S, Engelbrecht A. A cross-sectional survey of burnout amongst doctors in a cohort of public sector emergency centres in Gauteng, South Africa. Afr J Emerg Med 2018;8(3):95-99. https://doi. org/10.1016/j.afjem.2018.04.001

15. Van der Walt N, Scribante J, Perrie H. Burnout among anaesthetists in South Africa. South Afr Anaesth Analg 2015;21(6):169-172. https://doi.org/10.1080/22201181.2015.1102798

16. Balie GM. Burnout amongst obstetrics and gynaecology registrars in teaching hospitals for the University of the Witwaterrsand Medical School. Presented at the SAMA Conference 2018, University of the Witwatersrand Medical School. Presented at the SAMA Conference 2018,
Sun City, 17 - 19 August 2018. https://www.samedical.org/files/conference presentations/2018/ PRESENTATION_43.pdf (accessed 26 July 2019).

17. FluidSurveys Team. Response rate statistics for online surveys - what numbers should you be aimin for? Fluidsurveys 2014. http://fluidsurveys.com/university/response-rate-statistics-online-surveysaiming/ (accessed 26 July 2019).

18. Maslach C, Jackson SE, Leiter MP, et al. Maslach Burnout Inventory Manual, General Survey, Human Services Survey, Educators Survey and Scoring Guides. USA: Mind Garden Inc., 1996.

19. Doulougeri K, Georganta K, Montgomery A. 'Diagnosing' burnout among healthcare professionals: Can we find consensus? Cogent Medicine 2016;3(1):1237605. https://doi.org/10.1080/233120 5X.2016.1237605

20. Nedrow A, Steckler NA, Hardman I. Physician resilience and burnout: Can you make the switch? Fam Pract Manag 2013;20(1):25-30.

21. Ashkar K, Romani M, Musharrafieh U, et al. Prevalence of burnout syndrome among medical residents: Experience of a developing country. Postgrad Med J 2010;86(1015):266-271. https://doi. org/10.1136/pgmj.2009.092106

22. Stoddard JJ, Hargraves JL, Reed M, Vratil A. Managed care, professional autonomy, and income: Effects on physician career satisfaction. J Gen Intern Med 2001;16(10):675-684. https://doi.org/10.1111/ j.1525-1497.2001.01206.x

23. Burton A, Burgess C, Dean S, et al. How effective are mindfulness-based interventions for reducing stress among healthcare professionals? A systematic review and meta-analysis. Stress Health 2017;33(1):3-13. https://doi.org/10.1002/smi.2673

24. Van Vendeloo SN, Brand PLP, Verheyen CCPM. Burnout and quality of life among orthopaedic trainees in a modern educational programme: Importance of the learning climate. Bone Joint 2014;96-B(8):1133-1138. https://doi.org/10.1302/0301-620X.96B8.33609 